\title{
Correction to: Identifying Parkinson's disease with mild cognitive impairment by using combined MR imaging and electroencephalogram
}

\author{
Jiahui Zhang ${ }^{1,2} \cdot$ Yuyuan $\mathrm{Gao}^{2} \cdot$ Xuetao $\mathrm{He}^{3}$. Shujun Feng ${ }^{2}$. Jinlong $\mathrm{Hu}^{4} \cdot$ Qingxi Zhang $^{2}$ • Jiehao Zhao ${ }^{2}$. \\ Zhiheng Huang ${ }^{2} \cdot$ Limin Wang $^{2} \cdot$ Guixian $\mathrm{Ma}^{2} \cdot$ Yuhu Zhang ${ }^{2} \cdot \mathrm{Kun} \mathrm{Nie}^{2} \cdot$ Lijuan Wang $^{1,2}$ (1)
}

Published online: 23 July 2021

(C) European Society of Radiology 2021

\section{Correction to: European Radiology \\ https://doi.org/10.1007/s00330-020-07575-1}

The original version of this article, published on 03 January 2021, unfortunately contained two mistakes. The following corrections have therefore been made in the original: Firstly, the information was missing that Jiahui Zhang and Yuyuan Gao are co-first authors and that Kun Nie and Lijuan Wang are co-corresponding authors. Secondly, the presentation of Fig. 2 was incorrect. The corrected figure is given below. The original article has been corrected.

The online version of the original article can be found at https://doi.org/10. 1007/s00330-020-07575-1

\section{Kun Nie}

nk20080911@126.com

Lijuan Wang

wljgd68@163.com

1 The Second School of Clinical Medicine, Southern Medical University, No.1023, South Shatai Road, Baiyun District, Guangzhou 510515, China
2 Department of Neurology, Guangdong Provincial People's Hospital, Guangdong Academy of Medical Sciences, Guangdong Neuroscience Institute, No.106, Zhongshan 2nd Road, Yuexiu District, Guangzhou 510080, China

3 Department of Neuroelectrophysiology, Guangdong Provincial People's Hospital, Guangdong Academy of Medical Sciences, Guangzhou 510080, China

4 Communication and Computer Network Lab of Guangdong, School of Computer Science and Engineering, South China University of Technology, Guangzhou 510641, China 


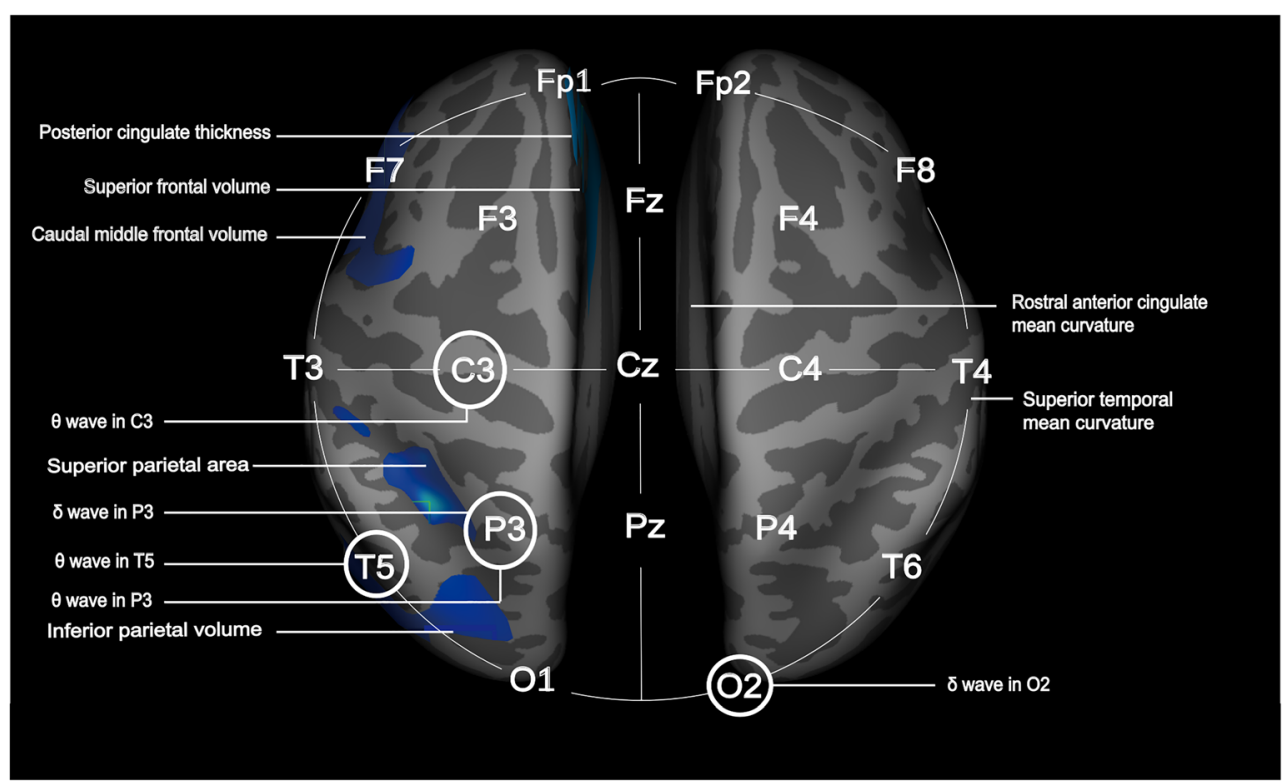

Fig. 2 Quantitative EEG and structure MR abnormality in PD-MCI

Publisher's Note Springer Nature remains neutral with regard to jurisdictional claims in published maps and institutional affiliations. 